\title{
Comprehensive analysis of RET common and rare variants in a series of Spanish Hirschsprung patients confirms a synergistic effect of both kinds of events
}

\author{
Rocio Núñez-Torres ${ }^{1,2}$, Raquel M Fernández ${ }^{1,2}$, Manuel Jesus Acosta ${ }^{1,2}$, Maria del Valle Enguix-Riego $0^{1,2}$,
} Martina Marbáa ${ }^{3}$ Juan Carlos de Agustín ${ }^{4}$, Luis Castaño ${ }^{5}$, Guillermo Antiñolo ${ }^{1,2}$ and Salud Borrego $0^{1,2^{*}}$

\begin{abstract}
Background: RET is the major gene associated to Hirschsprung disease (HSCR) with differential contributions of its rare and common, coding and noncoding mutations to the multifactorial nature of this pathology. In the present study, we have performed a comprehensive study of our HSCR series evaluating the involvement of both RET rare variants (RVs) and common variants (CVs) in the context of the disease.
\end{abstract}

Methods: RET mutational screening was performed by $\mathrm{dHPLC}$ and direct sequencing for the identification of RVs. In addition Taqman technology was applied for the genotyping of 3 RET CVs previously associated to HSCR, including a variant lying in an enhancer domain within RET intron 1 (rs2435357). Statistical analyses were performed using the SPSS v.17.0 to analyze the distribution of the variants.

Results: Our results confirm the strongest association to HSCR for the "enhancer" variant, and demonstrate a significantly higher impact of it in male versus female patients. Integration of the RET RVs and CVs analysis showed that in $91.66 \%$ of cases with both kinds of mutational events, the enhancer allele is in trans with the allele bearing the RET RV.

Conclusions: A gender effect exists on both the transmission and distribution of rare coding and common HSCR causing mutations. In addition, these RET CVs and RVs seem to act in a synergistic way leading to HSCR

phenotype.

\section{Background}

Hirschsprung disease (HSCR, OMIM \#142623) is a congenital malformation of the hindgut produced by a disruption in the neural crest cells (NCC) migration during embryonic development. This disorder results in an absence of intramural ganglion cells in the submucosal and myenteric plexuses producing a functional intestinal obstruction. According to the aganglionic segment length, patients could be classified as short-segment HSCR (S-HSCR), when aganglionosis extend as far as the rectosigmoid junction, and long-segment HSCR (L-

\footnotetext{
* Correspondence: salud.borrego.sspa@juntadeandalucia.es

'Unidad de Gestión Clínica de Genética, Reproducción y Medicina Fetal. Instituto de Biomedicina de Sevilla (IBIS), Hospital Universitario Virgen del Rocío/CSIC/Universidad de Sevilla, Sevilla, Spain

Full list of author information is available at the end of the article
}

HSCR), when it extends beyond it. HSCR presents an estimated incidence of $1 / 5000$ live births with sexdependent penetrance and male predominance of $4: 1$ $[1,2]$. It most commonly presents sporadically, although it can also be familial (up to $20 \%$ of the cases). The disease usually presents as isolated, though $30 \%$ of the cases are associated with chromosomal abnormalities, neurodevelopmental disorders and a variety of additional isolated anomalies and syndromes [2].

HSCR has a complex genetic etiology with several genes being described as associated with either isolated or syndromic forms. These genes encode for receptors, ligands (especially those participating in the RET and $E D N R B$ signaling transduction pathways), transcriptional factors or other cell elements that are usually involved

\section{Ciomed Central}


in the NCC development and migration that give rise to the enteric nervous system (ENS). Nevertheless, the RET proto-oncogene (OMIM +164761) is considered the major disease causing gene in HSCR [2].

RET has been extensively studied in HSCR patients and over 100 mutations have been identified along the gene (see HGMD). However, mutations in the RET coding sequence (CDS) account for only up to $50 \%$ or 7 $20 \%$ of familial and sporadic cases, respectively [2]. The involvement of RET in the pathogenesis of HSCR is further supported by the existence of a specific haplotype, constituted by common RET polymorphisms, which seems to be responsible for the majority of sporadic forms [3,4]. This HSCR-associated RET haplotype is characterized by a common allele (c.73+9277T, rs2435357) within a conserved enhancer-like sequence in intron 1 (MCS+9.7) [3,5], making a 20-fold greater contribution to risk than coding mutations do [3]. It has been demonstrated a difference in ability of SOX10 to bind to MCS+9.7 and transactivate RET depending on the bearing allele at such specific locus [5]. Recently, one SNP located closed to rs2435357 and in complete linkage disequilibrium with it $(c .73+9494 \mathrm{~A}>\mathrm{C}$, rs2506004) has been identified as a binding site for NXF/ARNT2 and SIM2-ARNT2 that modifies RET expression, demonstrating that more than one SNP can influence gene expression and ultimately HSCR phenotype [6]. In this way, the combination of common variants $(\mathrm{CV})$, such as the so-called enhancer variants, and rare variants (RV), such as RET CDS mutations, seems to explain in part the complexity of HSCR.

In the present study, we have evaluated the implication of both RET CVs and RVs in our whole cohort of 282 Spanish patients. In order to elucidate the molecular basis of the sex-dependent difference in HSCR we sought to perform a case-control study to analyze the distribution of the RET variants based in gender. In addition, we have performed a segregation analysis in patients for both RVs and CVs, with the aim to clarify their contribution to HSCR phenotype.

\section{Methods}

\section{Patients and Control Subjects}

In the mutational screening we have included an extension of our previously described cohort [7] incorporating a total of 176 new Spanish HSCR patients. 170 of these patients were sporadic cases, 6 were familial belonging to 3 different families. Taking together all the data, our cohort is constituted by a total of 282 HSCR patients (253 sporadic and 29 familial cases belonging to 16 families). According to the length of the aganglionic segment, the patients were catalogued as S-HSCR $(55,08 \%)$, L-HSCR $(11,58 \%)$ and Total Colonic Aganglionosis (TCA) (5,61\%), while this information was not available for $27,73 \%$ of HSCR patients. Male:female ratios were $4: 1$ and 3:1 for short and long forms (including the TCA patients), respectively. $4,6 \%$ of our HSCR patients were syndromic cases with Down syndrome (6 cases), Waardenburg syndrome (2 cases), Ondine course (1 case), cardiopathy (2 cases) or encephalopathy ( 2 cases). However due to incomplete clinical data of part of our patients, our percentage might be underestimated, taking into account that the overall percentage reported for syndromic HSCR patients is around 18\% [2].

Genotyping analysis of RET CVs was performed exclusively in the 253 sporadic cases of our cohort, since previous studies suggest that CVs have a minor effect in familial cases, being those cases more probably associated to CDS mutations in RET or other secondary genes [2]. 103 of our sporadic cases had been already evaluated in a previous study [8], thus we have genotyped the remaining 150 sporadic cases, as well as their available parents. Trios necessary for the Transmission Disequilibrium Test (TDT) analysis could be completed in 134 of the 150 new families.

A total of 178 control individuals (129 males and 49 females) comprising unselected, unrelated, race, age, and sex-matched individuals were included in this study. All the controls were healthy voluntary donors, who came to the Hospital for other reasons and did not present any symptom suggestive of HSCR.

An informed consent was obtained from all the participants for clinical and molecular genetic studies. The study was approved by the Ethics Committee for clinical research in the Hospital Universitario Virgen del Rocio of Seville, and complies with the tenets of the declaration of Helsinki.

\section{PCR, dHPLC and Sequence analysis}

We screened all the RET exons, including intron/exon boundaries by PCR-dHPLC as previously described [7]. The exons of those patients with aberrant wave profiles were subjected to sequence analysis as previously reported [9]. When a novel RET variant was identified in a proband, all the available family members were analyzed for the appropriate exon in order to perform the corresponding segregation analysis.

\section{Genotyping of RET SNPs and statistical analysis}

We sought to analyze the distribution of three $R E T$ SNPs (rs2435357 (c.73+9277T > C), rs2505532 (c.74$1370 \mathrm{C}>\mathrm{T}$ ) and rs2565206 (c.74-126G > T)) in HSCR patients to compare with that of the controls obtained in our previous studies $[4,8]$. Large scale genotyping of all these RET SNPs was performed using TaqMan-based techniques for allelic discrimination (TaqMan ${ }^{\circledR}$ SNP Genotyping Assay, Applied Biosystems, Foster City, CA) 
according to the manufacturer's recommendations and described elsewhere [10].

For each variant, Hardy-Weinberg equilibrium was verified in both the control and patients groups. Allelic and genotypic frequencies of the 3 RET SNPs were calculated and then compared between patients and controls. We also compared transmitted versus non-transmitted alleles from unaffected parents to affected offspring for each locus. On the other hand, RET haplotypes were constructed as previously described $[4,8]$, and their distribution was compared between patients and controls as well as between transmitted and non-transmitted chromosomes in the context of the HSCR trios. Furthermore, allelic, genotypic and haplotypic distributions were compared between cases and controls in different subsets based in gender (male patients versus male controls, and female patients versus female controls). Despite the fact that the group of male patients is higher that the group of females, each subset of patients was compared to a group of sexmatched controls similar in size. All the comparisons were analysed using $\chi^{2}$ test with Yate's correction, considering statistical significance at $\mathrm{p}<0.05$ and 2 degrees of freedom (SPSS v.17.0 and R-software). For each variant we calculated the OR $(95 \% \mathrm{CI})$ for the recessive, dominant and additive models (unadjusted and adjusted for gender) [11], and the generalized OR (GOR) (95\% CI) [12].

\section{Results}

Identification of RET CDS mutations

In order to determine the mutational status of our whole cohort of 282 HSCR patients, we developed a mutational screening by dHPLC of the 176 cases that had not been analyzed yet. RET mutational status of remaining 106 cases was available from a previous study [7].

We have detected 16 different germline mutations in a total of 20 sporadic cases, all of them in heterozygosis (Table 1, Figure 1). 13 of the 16 mutations are novel and all of them, except for A156S, are predicted to be probably damaging variants using physical and comparative considerations http://genetics.bwh.harvard.edu/pph/ and http://blocks.fhcrc.org/sift/SIFT.html. Moreover, none of them were detected in the control population tested. 10/ 18 mutations are maternally inherited while inheritance from paternal origin occurs with a similar frequency than the de novo events (4/18 and 4/18 respectively). With this new data, our complete cohort of 282 HSCR patients presents a $R E T$ mutational frequency of $18.75 \%$ in familial cases ( 3 CDS mutations in 16 families screened) and $11.11 \%$ in non familial cases (29 CDS mutations in the 253 sporadic patients screened).

\section{Genotyping of RET SNPs}

We have analyzed allelic and genotypic distribution of 3 RET SNPs for the new 150 sporadic HSCR cases not previously tested $[4,8]$ and for 178 controls individuals. Allelic distributions for rs2435357 and rs2505532 showed similar statistical differences in cases vs controls than previously observed $\left(\chi^{2}=135.41 \mathrm{p}<<10^{-6}\right.$ and $\chi$ $=96.45, \mathrm{p}<<10^{-6}$ respectively), $[4,8]$. No statistical difference was found for rs2565206, although a slight trend towards significance was observed $(\chi=3.62, \mathrm{p}=$ $0.0569263)$. However, when we performed this analysis, including not only the new cases but the whole series of sporadic patients, the difference in the distribution of this variant in cases vs controls get statistical significance $\left(\chi^{2}=6.74, p=0.0094106\right)$. Haplotypic distribution also resulted statistically different when comparing HSCR patients with controls $\left(\chi^{2}=159.62 \mathrm{p}<<10^{-6}\right)$. Moreover, TDT analysis performed for the 134 HSCR trios showed a statistical difference when comparing transmitted haplotypes versus non transmitted haplotypes $\left(\chi^{2}=87.82 \mathrm{p}<<10^{-6}\right)$.

On other hand, we have performed an additional statistical analysis comparing allelic, genotypic and haplotypic distribution in different subsets of patients and controls classified by gender (male patients vs male controls, and female patients vs female controls). Allelic distribution of rs2435357 and rs2505532 was clearly different in males and females HSCR patients compared with the corresponding control individuals, but presenting a considerably higher difference in male cases $(23$ orders of magnitude larger in males than in females, Table 2, Figure 2, Additional File 1). Regarding the rs2565206 variant, its distribution was found to be significantly different in male cases versus male controls whereas no difference was observed in female patients compared with female controls. Similar results were obtained for the analysis of the genotypic distribution. Haplotypic distribution was also different for both sets, although distribution was perceptibly more different in males (males: $\chi^{2}=167.6938, \mathrm{p}=2.26^{*} 10^{-34}$ females: $\chi=$ 49.17491, $\left.\mathrm{p}=2.04^{*} 10^{-9}\right)$. TDT applied to haplotypes was also performed in male and female subsets and resulted in an unequal transmission as we expected (males: $\chi=$ 114.5463, $\mathrm{p}=4.47^{*} 10^{-23}$; females: $\chi=42.91487, \mathrm{p}=$ $\left.3.84 * 10^{-8}\right)$.

\section{Analysis of the relative position of RET CDS mutations and the enhancer variant}

Both the genotyping of RET CVs and mutational screening of RET RVs was completed for 253 sporadic HSCR patients. Of them, 34 (13\%) cases presented neither RET RVs nor the enhancer variant, 192 (76\%) harbored only the enhancer variant in either heterozygosis or homozygosis, 13 (5\%) had exclusively a RET RV (CDS), and 14 (6\%) presented with both disease allelic types. The frequency distributions of these four mutational classes classified according to the gender presents similar 
Table 1 RET germline mutations identified in our series of HSCR patients

\begin{tabular}{|c|c|c|c|c|c|c|c|}
\hline Patient & $\begin{array}{l}\text { Exon/ } \\
\text { Intron }\end{array}$ & $\begin{array}{l}\text { Nucleotide } \\
\text { change }\end{array}$ & $\begin{array}{l}\text { Amino acid } \\
\text { change }\end{array}$ & $\begin{array}{c}\text { Parent origin of the } \\
\text { mutation }\end{array}$ & Gender & $\begin{array}{c}\text { Length of aganglionic } \\
\text { segment }\end{array}$ & Ref. \\
\hline HSCR 193 & 2 & c. $287 \mathrm{~A}>\mathrm{C}$ & p.Tyr96Ser & Mother & Female & Not Available & - \\
\hline HSCR 133 & 3 & c. 466 G > T & p.Ala156Ser & De novo & Female & $\mathrm{S}-\mathrm{HSCR}$ & - \\
\hline HSCR 166 & 5 & $c .937 \mathrm{C}>\mathrm{T}$ & p.Arg313Trp & Mother & Male & Not Available & - \\
\hline $\begin{array}{l}\text { HSCR } \\
\text { V135 }\end{array}$ & 5 & c.988insC & p.R330PfsX353 & Mother & Male & S-HSCR & - \\
\hline HSCR 223 & 5 & c.1042 C > T & p.Arg348Trp & Mother & Male & Not Available & - \\
\hline HSCR 109 & 6 & C.1118 C > T & p.Ala373Val & Father & Female & $\mathrm{S}-\mathrm{HSCR}$ & [7] \\
\hline HSCR 217 & 6 & C.1118 C > T & p.Ala373Val & Mother & Male & S-HSCR & [7] \\
\hline HSCR 220 & 6 & C.1118 C > T & p.Ala373Val & Mother & Male & $\mathrm{S}-\mathrm{HSCR}$ & [7] \\
\hline HSCR 99 & 7 & c. $1267 \mathrm{G}>\mathrm{A}$ & p.Gly423Arg & Mother & Female & S-HSCR & - \\
\hline HSCR V67 & 7 & C. $1325 T>C$ & p. Leu442Pro & Mother & Male & Not Available & - \\
\hline HSCR 187 & 8 & c.1627 T > A & p.Trp543Arg & De novo & Female & S-HSCR & - \\
\hline HSCR 260 & 10 & c. $1859 \mathrm{G}>\mathrm{A}$ & p.Cys620Tyr & Father & Male & L-HSCR & - \\
\hline HSCR V24 & 14 & $c .2459 G>A$ & p. Arg $820 \mathrm{His}$ & De novo & Male & Not Available & - \\
\hline HSCR 204 & 17 & c.2858 C > T & p.Pro953Leu & Mother & Male & $\mathrm{S}-\mathrm{HSCR}$ & - \\
\hline HSCR 278 & 18 & c.2944 C > T & p.Arg982Cys & Not Available & Male & S-HSCR & [19] \\
\hline HSCR V22 & 18 & c.2944 C > T & p.Arg982Cys & Father & Male & Not Available & [19] \\
\hline HSCR 15 & 18 & c. 2975 C > T & p.Pro992Leu & De novo & Male & L-HSCR & - \\
\hline HSCR 151 & 19 & C.3185 A > G & p.Tyr1062Cys & Mother & Female & $\mathrm{S}-\mathrm{HSCR}$ & [20] \\
\hline HSCR 198 & 19 & c.3185 A > G & p.Tyr1062Cys & Father & Male & Not Available & {$[20]$} \\
\hline HSCR 278 & 20 & $c^{*} 4$ delTCTTinsAAA & & NA & Male & $\mathrm{S}-\mathrm{HSCR}$ & - \\
\hline
\end{tabular}

results. Examining the patients with both kinds of mutational events, segregation analysis let us to observe that $91.66 \%$ of cases (11 of 12 patients) harbors the "enhancer mutation" in trans with respect the allele bearing the RET RV. The remaining patient was homozygous for the enhancer variant.

Of the 34 HSCR patients with no RET CDS mutation and no enhancer variation, 11 presented another kind of mutational events related with HSCR: 1 chromosomal translocation ( $\mathrm{t}(10: 22))$, 6 chromosomal 21 triplication and 4 mutations in other HSCR genes (EDNRB, GDNF, and NTF3) $[7,13,14]$.

\section{Discussion}

Previous genome-wide linkage/association studies have identified several modifier genes in different genomic regions, but $R E T$ is the only gene known to play a major role in all forms of HSCR susceptibility and a

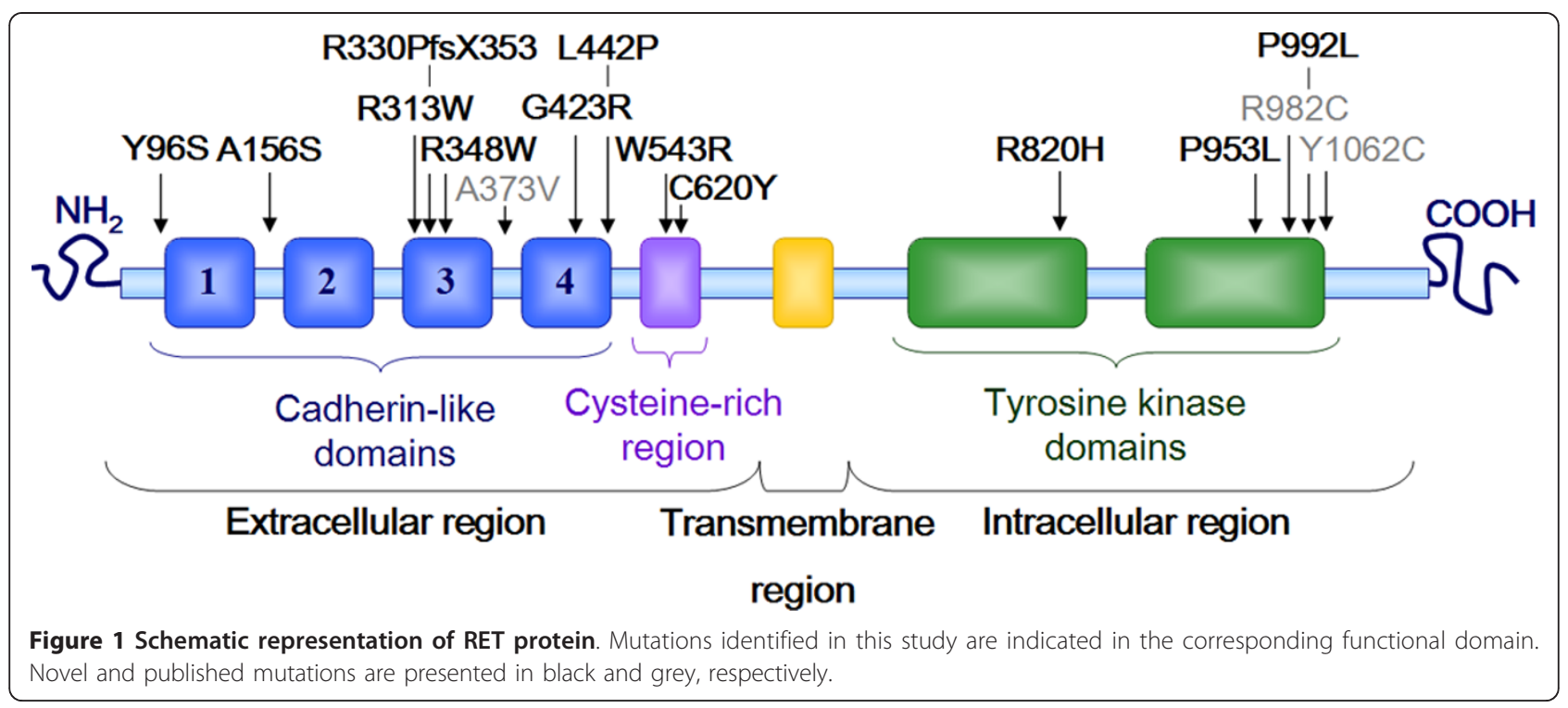


Table 2 Allelic distribution frequency of the RET SNPs

\begin{tabular}{|c|c|c|c|c|c|}
\hline \multirow[b]{2}{*}{$R E T$ variants } & \multirow[b]{2}{*}{ Alleles } & \multicolumn{2}{|c|}{ Male } & \multicolumn{2}{|c|}{ female } \\
\hline & & HSCR (\%) & Controls (\%) & HSCR (\%) & Controls (\%) \\
\hline c.73+9277T > C & $T$ & $250(64.77)$ & $52(20.15)$ & $37(56.06)$ & $21(21.43)$ \\
\hline \multirow[t]{2}{*}{ rs2435357 } & C & $136(35.24)$ & $206(79.85)$ & 29 (43.93) & $77(78.57)$ \\
\hline & & \multicolumn{2}{|c|}{$\chi^{2}=121.79 \mathrm{p}=2.55 \cdot 10^{-28} \Re$} & \multicolumn{2}{|c|}{$\chi^{2}=36.96 \mathrm{p}=0.0000117 \Re$} \\
\hline c.74-1370C > T & C & $328(84.97)$ & $143(55.43)$ & $56(84.85)$ & $46(46.94)$ \\
\hline \multirow[t]{2}{*}{ rs2505532 } & T & $58(15.03)$ & $115(44.57)$ & $10(15.15)$ & $52(53.06)$ \\
\hline & & \multicolumn{2}{|c|}{$\chi^{2}=67.22 p=2.42 \cdot 10^{-16} \Re$} & \multicolumn{2}{|c|}{$\chi^{2}=22.52 p=0.0000021 \Re$} \\
\hline c.74-126G > T & G & $300(77.72)$ & $175(67.83)$ & $48(72.72)$ & $70(71.42)$ \\
\hline \multirow[t]{2}{*}{ rs2565206 } & T & $86(22.28)$ & $83(32.17)$ & $18(27.27)$ & $28(28.57)$ \\
\hline & & \multicolumn{2}{|c|}{$\chi^{2}=7.31 \mathrm{p}=0.0068453 \Re$} & \multicolumn{2}{|c|}{$\chi^{2}=0.00 p=0.9965511$} \\
\hline
\end{tabular}

quantitative study of its allelic spectrum should provide clues on the role of both rare and common sequence variants in the complex inheritance of HSCR, particularly its relationships to factors that are correlated with risk, such as gender.

After our RET CDS mutational screening, we have found that our whole series of Spanish HSCR patients presents a mutational frequency of $11.11 \%$ in sporadic cases and $18.75 \%$ in familial ones, which is concordant with previous studies that report values of up to $50 \%$ in familial forms and 7-20\% in sporadic cases [2].

Genotyping analysis of RET common SNPs shows similar results to those previously described, confirming thereby the prominent role of either the intronic enhancer mutation (rs2435357, [3]) or the RET "risk haplotype" $[4,8]$ in the pathogenesis of sporadic HSCR.

As previously commented, one of the major features of HSCR, especially in the short-segment forms, is the sex-dependent penetrance and male predominance of $4: 1$ [1]. Nevertheless the reason of this sex difference is still unclear. It has been postulated that sex differences could arise from mutations on the $\times$ chromosome, but genome-wide mapping studies have failed to identify an $\mathrm{X}$-linked gene with a relevant impact per se in HSCR.

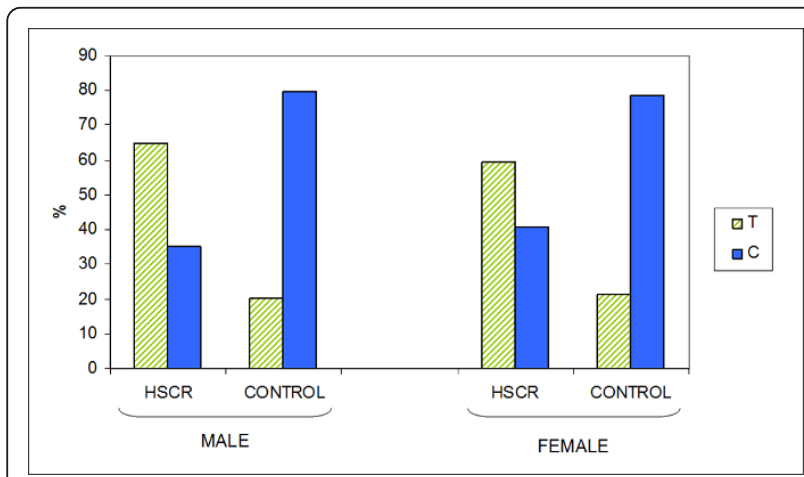

Figure 2 Allelic distribution frequency of RET enhancer variant rs2435357 in Male and Female subset.
Previous studies have indeed demonstrated differences in the transmission frequency of the "enhancer mutation" (rs2435357) depending on the offspring gender or parent gender $[3,5]$. For this reason, we sought to perform an additional case-control study to analyze the distribution of the RET variants based in gender. We found a different allelic distribution between HSCR cases and controls in both, males and females, for rs2435357 and rs2505532. However and noteworthy, significant differences in HSCR versus controls in the male subset were considerably increased with respect the female subset, which is concordant with the sex-biased transmission of the rs2435357 variant published by Emison et al. Nevertheless, no significant differences were found when comparing males vs females, in agreement with the similarity in transmission frequency by gender after applying TDT reported by Emison et al., 2010 [5]. The same phenomenon was observed for the haplotype analysis, which as a whole is consistent with the greater incidence in males than in females. However we cannot discard the possibility that the difference in the sample size of the corresponding subsets might be interfering to some extent in the results observed.

On the other hand, the association of rs2565206 to HSCR has been found to be sex-dependent and restricted to males. Probably, for this reason this SNP presented association to HSCR only when we extended our cohort, since we increased considerably the proportion of male HSCR cases. It is relevant to note that this specific variant has also been previously proposed to have a role in the pathogenesis of sporadic medullary thyroid cancer, since an over-representation of the $\mathrm{T}$ allele was found in different studies reported $[8,15,16]$. Although the most accepted hypothesis to explain these associations is the linkage with a still unidentified functional locus within or nearby $R E T$, it has been proposed as well that this variant might slightly modulate per se the expression of the RET proto-oncogene, given that a new binding motif is created for NAFT transcription factor in the presence of the $\mathrm{T}$ allele [8]. The restriction 
to association of the $\mathrm{G}$ allele to the male subset of HSCR patients could be therefore considered as another hallmark of the complex nature of Hirschsprung disease.

After the evaluation of RVs and CVs in our HSCR series, we have classified our cohort of patients according to RET mutational status in 4 subsets (CDS mutation only, enhancer mutation only, both or any of those events) obtaining similar frequencies in these subsets than Emison et al [5]. Nevertheless, taking a further step in this study we performed a complete segregation analysis in patients with both RV and CV. $91.66 \%$ of cases with both mutational events resulted to present the enhancer variant in trans with regard to the CDS mutation. It supports that the CVs may act as modifier alleles of that bearing the RV, as speculated by Emison et al [5]. In this way, we could be talking about a synergy effect between two alleles, which is in agreement with the additive/multiplicative model proposed for HSCR.

On the other hand, in our cohort, we have detected a 13\% of cases with no RET RVs or CVs. It has been previously proposed that mutations in other HSCR genes could be responsible for the HSCR phenotype in those patients, but the analysis of other HSCR genes and chromosomal alterations can only explain $31 \%$ of these cases. Alternatively, $R E T$ deletions have been also proposed as a possible underlying molecular mechanism [5], although our MLPA analysis revealed the absence of this kind of mutational event affecting RET or other HSCR genes [17,18]. Nevertheless, we cannot discard the presence of deletions in non-coding regulatory regions of RET or other HSCR genes which may lead to a loss-of-function of the protein.

\section{Conclusions}

In summary, from our whole results we could extract several conclusions: (1) A gender effect exists on both the transmission and distribution of rare coding and common HSCR causing mutations (2) The variant rs 2565206 has been found to be associated to HSCR in a sex-dependent manner, being such association restricted to male patients (3) RET CVs and RVs seem to act in a synergistic way leading to HSCR phenotype (4) A portion of HSCR cases without RET CVs and RVs might be explained by mutational events in another still unidentified HSCR loci, and efforts should be made to propose alternative methodological approaches that could lead to the elucidation of their molecular causes.

\section{Additional material}

Additional File 1: Calculations of OR and GOR values. OR ( $95 \% \mathrm{Cl})$ and the generalized $O R(G O R)(95 \% \mathrm{Cl})$ were calculated for the recessive, dominant and additive models for each variant (unadjusted and adjusted for gender).

\section{Acknowledgements}

We would like to thank the family that participated in this study. This study was funded by Fondo de Investigación Sanitaria, Spain (PI070080, PI1001290, and PI071315 for the E-Rare Project), Consejeria de Innovación Ciencia y Empresa de la Junta de Andalucia (CTS-2590), and Consejeria de Salud de la Junta de Andalucia (PI0249-2008). The CIBER de Enfermedades Raras is an initiative of the ISCIII.

\section{Author details}

${ }^{1}$ Unidad de Gestión Clínica de Genética, Reproducción y Medicina Fetal. Instituto de Biomedicina de Sevilla (IBIS), Hospital Universitario Virgen del Rocío/CSIC/Universidad de Sevilla, Sevilla, Spain. ${ }^{2}$ Centro de Investigación Biomédica en Red de Enfermedades Raras (CIBERER), Sevilla, Spain.

${ }^{3}$ Departamento de Genómica y Bioinformática. Centro de Investigación Príncipe Felipe (CIPF), Valencia, Spain. ${ }^{4}$ Unidad de Gestión Clínica de Cirugía Infantil, Hospital Universitario Virgen del Rocío, Sevilla, Spain. ${ }^{5}$ Grupo de investigación en Endocrinología y Diabetes, Hospital de Cruces, Vizcaya, Spain.

\section{Authors' contributions}

SB coordinated all the studies here presented. JCA and LC recruited the HSCR patients and provided clinical information. RMF and SB developed the design of the experiments. RN-T, MJA and MVE-R carried out the molecular genetic analyses. MB performed the statistical tests. RN-T and SB drafted the manuscript. RMF and GA helped to draft the manuscript. All authors have read and approved the final manuscript.

\section{Competing interests}

The authors declare that they have no competing interests.

Received: 17 November 2010 Accepted: 13 October 2011 Published: 13 October 2011

\section{References}

1. Chakravarti A, Lyonnet S: Hirschsprung Disease. In The metabolic and molecular bases of inherited disease. Volume 2002. Edited by: Scriver CS. McGraw-Hill; 2002:6231-6255.

2. Amiel J, Sproat-Emison E, Garcia-Barcelo M, Lantieri F, Burzynski G, Borrego S, Pelet A, Arnold S, Miao X, Griseri P, Brooks AS, Antinolo G, de Pontual L, Clement-Ziza M, Munnich A, Kashuk C, West K, Wong KK, Lyonnet S, Chakravarti A, Tam PK, Ceccherini I, Hofstra RM, Fernandez R, Hirschsprung Disease Consortium: Hirschsprung disease, associated syndromes and genetics: a review. J Med Genet 2008, 45:1-14.

3. Emison ES, McCallion AS, Kashuk CS, Bush RT, Grice E, Lin S, Portnoy ME, Cutler DJ, Green ED, Chakravarti A: A common sex-dependent mutation in a RET enhancer underlies Hirschsprung disease risk. Nature 2005, 434:857-863.

4. Fernandez RM, Boru G, Pecina A, Jones K, Lopez-Alonso M, Antiñolo G, Borrego S, Eng C: Ancestral RET haplotype associated with Hirschsprung's disease shows linkage disequilibrium breakpoint at -1249 . J Med Genet 2005, 42:322-327.

5. Emison ES, Garcia-Barcelo M, Grice EA, Lantieri F, Amiel J, Burzynski G, Fernandez RM, Hao L, Kashuk C, West K, Miao X, Tam PK, Griseri P, Ceccherini I, Pelet A, Jannot AS, de Pontual L, Henrion-Caude A, Lyonnet S, Verheij JB, Hofstra RM, Antiñolo G, Borrego S, McCallion AS, Chakravarti A: Differential contributions of rare and common, coding and noncoding Ret mutations to multifactorial Hirschsprung disease liability. Am J Hum Genet 2010, 87(1):60-74.

6. Sribudiani Y, Metzger M, Osinga J, Rey A, Burns AJ, Thapar N, Hofstra RM: Variants in RET associated with Hirschsprung disease affect binding of transcription factors and gene expression. Gastroenterology 2011, 140(2):572-582, e2.

7. Ruiz-Ferrer F, Fernandez R, Antiñolo G, López- Alonso M, Eng C, Borrego S: A complex additive of inheritance for Hirschprung disease (HSCR) is supported by both RET mutations and predisposing RET haplotypes. Genet Med 2006, 8:1-6.

8. Borrego S, Wright FA, Fernandez RM, Williams N, Lopez-Alonso M, Davuluri R, Antiñolo G, Eng C: A founding locus within the RET protooncogene may account for a large proportion of apparently sporadic Hirschsprung disease and a subset of cases of sporadic medullary thyroid carcinoma. Am J Hum Genet 2003, 72:88-100. 
9. Sánchez-Mejías A, Fernández RM, López-Alonso M, Antiñolo G, Borrego S: New roles of EDNRB and EDN3 in the pathogenesis of Hirschsprung disease. Genet Med 2010, 12:39-43.

10. Fernández RM, Sánchez-Mejías A, Ruiz-Ferrer M, López-Alonso M, Antiñolo G, Borrego S: Is the RET proto-oncogene involved in the pathogenesis of intestinal neuronal dysplasia type B? Mol Med Rep 2009, 2:265-270.

11. Zintzaras $E$, Lau J: Synthesis of genetic association studies for pertinent gene-disease associations requires appropriate methodological and statistical approaches. J Clin Epidemiol 2008, 61:634-45.

12. Zintzaras E: The generalized odds ratio as a measure of genetic risk effect in the analysis and meta-analysis of association studies. Stat Appl Genet Mol Biol 2010, 9:Article21.

13. Ruiz-Ferrer M, Fernandez RM, Antiñolo G, Lopez-Alonso M, Borrego S: NTF3 , a gene involved in the enteric nervous system development, as a candidate gene for Hirschsprung disease. J Pediatr Surg 2008, 43(7):1308-1311.

14. Ruiz-Ferrer M, Torroglosa A, Luzón-Toro B, Fernández RM, Antiñolo G, Mulligan LM, Borrego S: Novel mutations at RET ligands genes preventing receptor activation are associated to Hirschsprung's disease. J Mol Med 2011, 89(5):471-80.

15. Fernandez RM, Robledo M, Antinolo G, Pecina A, Ruiz-Llorente S, Eng C, Borrego S: The RET IVS1-126G > T variant is strongly associated with the development of sporadic medullary thyroid cancer. Thyroid 2004, 14(4):329-331.

16. Fernández RM, Peciña A, Antiñolo G, Navarro E, Borrego S: Analysis of RET polymorphisms and haplotypes in the context of sporadic medullary thyroid carcinoma. Thyroid 2006, 16(4):411-417.

17. Núñez-Torres R, Fernández RM, López-Alonso M, Antiñolo G, Borrego S: A novel study of copy number variations in Hirschsprung disease using the multiple ligation-dependent probe amplification (MLPA) technique. BMC Med Genet 2009, 10:119-124

18. Sánchez-Mejías A, Núñez-Torres R, Fernández RM, Antiñolo G, Borrego S: Novel MLPA procedure using self-designed probes allows comprehensive analysis for CNVs of the genes involved in Hirschsprung disease. BMC Med Genet 2010, 11:71-79.

19. Mulligan LM, Eng C, Attié T, Lyonnet S, Marsh DJ, Hyland VJ, Robinson BG, Frilling A, Verellen-Dumoulin C, Safar A, et al: Diverse phenotypes associated with exon 10 mutations of the RET proto-oncogene. Hum Mol Genet 1994, 3:2163-2167.

20. Wu TT, Tsai TW, Chu CT, Lee ZF, Hung CM, Su CC, Li SY, Hsieh M, Li C: Low RET mutation frequency and polymorphism analysis of the RET and EDNRB genes in patients with Hirschsprung disease in Taiwan. J Hum Genet 2005, 50(4):168-74

\section{Pre-publication history}

The pre-publication history for this paper can be accessed here: http://www.biomedcentral.com/1471-2350/12/138/prepub

doi:10.1186/1471-2350-12-138

Cite this article as: Núñez-Torres et al:: Comprehensive analysis of RET common and rare variants in a series of Spanish Hirschsprung patients confirms a synergistic effect of both kinds of events. BMC Medical Genetics 2011 12:138.

\section{Submit your next manuscript to BioMed Central and take full advantage of:}

- Convenient online submission

- Thorough peer review

- No space constraints or color figure charges

- Immediate publication on acceptance

- Inclusion in PubMed, CAS, Scopus and Google Scholar

- Research which is freely available for redistribution

Submit your manuscript at www.biomedcentral.com/submit 